\title{
Effects of red meat diet on gut microbiota in mice
}

\author{
Xiaoyan LIU ${ }^{1,2}$, Fang TAN ${ }^{1}$, Min CUI ${ }^{1}$, Danping $\mathrm{LI}^{1,2}$, Ping $\mathrm{YAO}^{1 \star}$ (1)
}

\begin{abstract}
We aimed to evaluate the effect of red meat diet on gut microbiota in mice. Balb/c mice at weaning were randomized into control group and red meat groups with different proportions (25\%,50\%, and 75\%). Mice were fed with a standard pellet diet as control group, while those were fed with different proportions of red meat diet as red meat groups. After 8 weeks, they were sacrificed and their intestinal contents were obtained for $16 \mathrm{~S}$ rRNA sequencing and bioinformatics analysis. Our results showed that there were significant-structural differences among the four groups. The top-two most abundant phylum were Firmicutes and Bacteroidetes. In the red meat groups, the abundance of Bacteroidetes was increased, but the abundance of Firmicutes was decreased. At the family level, Bacteroidaceae and Family XIII were significantly higher in the high-dose group than those in the control group. There were also significant differences in abundance of many genera. In conclusion, different proportions of red meat diet may lead to changes in gut microbial flora in mice. These changes may be pathological and may be related to the frequent occurrence of many diseases.
\end{abstract}

Keywords: red meat diet; $16 \mathrm{~S}$ rRNA sequencing; gut microbiome; mice; bioinformatics analysis

Practical Application: We evaluated the effect of red meat diet on gut microbiota in mice. We found that different proportions of red meat diet may lead to changes in gut microbial flora in mice. These changes may be pathological and may be related to the frequent occurrence of many diseases.

\section{Introduction}

The interaction between the gut microbiota and the host is crucial for maintaining homeostasis and body health (Jiang \& Schnabl, 2020). Microbiota facilitates carbohydrate digestion (Makki et al., 2018), bile acid metabolism (Wang et al., 2020), maintenance of the integrity of gut barrier against pathogen infection (Mendes et al., 2019), and vitamin synthesis (Yatsunenko et al., 2012). However, the gut microbiota is associated with the pathogenesis of many diseases, including Colon Cancer (Birt \& Phillips, 2014), Cardiovascular (Scarmozzino et al., 2020) and liver diseases. The composition of the gut microbiota may be affected by many factors in the host, including physiology, pathology, living environment, immune system and lifestyle (Butel et al., 2018). Among them, diet plays a significant role (Steer et al., 2000). Foods are mainly digested and absorbed in the stomach and small intestine, but a substantial quantity of foods may enter the large intestine and alter the diversity of gut bacteria (van Hylckama Vlieg et al., 2011; Rist et al., 2013).

It has revealed that fiber- and vegetable-rich diets and physical activity may contribute to reduced incidences of many diseases. However, the consumptions of red and processed meat or alcoholic beverages are related to elevated incidences of many diseases (Birt \& Phillips, 2014), such as colon cancer (Abu-Ghazaleh et al., 2021), inflammatory bowel disease (Li et al., 2020) and cardiovascular disease (Zhong et al., 2020). In humans, high consumption of red meat and reduced content of nondigestible carbohydrates in daily diet may increase the risks of inflammatory bowel disease and colorectal cancer (Hou eta al., 2011; Alexander \& Cushing
2011). It has been hypothesized that the reason may be due to the changes in colonic microflora (Ijssennagger et al., 2015; O'Keefe et al., 2015). Evidence shows that dietary intervention of the human gut microbiota is feasible and has been proven as efficacious in voluntary trials (Steer et al., 2000).

The relationship between microbiota and food is bidirectional. While the food in-take can alter the gut microbiota, the modified microbiota in turn produce a variety of metabolites, which could be beneficial or detrimental (Dey 2019). In mouse models of colitis, microbial colonization is required for the development of active inflammation (Hudcovic et al., 2001). Their composition and diversity will change with different diet (Wu et al., 2011). Therefore, it is possible to reduce the risk of developing colitis through controlled diet, which requires a profound understanding of the relationship between diet and the gut microbiota.

The Illumina instruments provide the highest yield and quality data currently (Zhang et al., 2018). Therefore, in this study, we aimed to evaluate the effect of red meat diet on gut microbiota in mice.

\section{Materials and methods}

\subsection{Samples}

The protocols were approved by the institutional ethical committee of Xinjiang Medical University. Different proportions of red meat diets were obtained from the Animal Experimental

${ }^{1}$ Department of Gastroenterology, The First Affiliated Hospital of Xinjiang Medical University, Xinshi District, Urumqi, Xinjiang Uygur Autonomous Region, P.R. China

${ }^{2}$ Department of Gastroenterology, The Fifth Affiliated Hospital of Xinjiang Medical University, Xinshi District, Urumqi, Xinjiang Uygur Autonomous Region, P.R. China

*Corresponding author: pingyaoyfy@163.com 
Center of Xinjiang Medical University. The proportions of red meat were $0 \%, 25 \%, 50 \%$, and $75 \%$, which were served as diets for control group, low-dose group, medium-dose group and high-dose group, respectively. All the specific configuration of red meat diet was shown in Table1.

A total of 24 healthy balb/c mice (aged 3 weeks; male and female each half; Certificate number, SCXK(XIN)2018-0002) were obtained from the Animal Experimental Center of Xinjiang Medical University (Xinjiang, China). The animals were housed in standard feeding environment. Mice were randomized into control group (mice number 1-1,1-2,1-3,1-4,1-5, and 1-6), low-dose group (mice number 2-1,2-2,2-3,2-4,2-5, and 2-6), medium-dose group (mice number 3-1,3-2,3-3,3-4,3-5, and 3-6) and high-dose group (mice number 4-1,4-2,4-3,4-4,4-5, and 4-6). The mice were provided with water and fed with ad libitum. After 8 weeks, all mice were sacrificed by cervical dislocation. The flowchart was shown in Figure 1.

\subsection{Sequencing}

Fecal samples were collected aseptically from the ileum, cecum and distal colon. Intestinal contents were obtained for further microbial community analysis. DNA samples were extracted using the E.Z.N.A. stool DNA Kit (Omega Bio-tek, Norcross, GA, U.S.) following the manufacturer's protocols. DNA samples were detected by $1 \%$ agarose gel electrophoresis. The V3-V4 region of the bacteria $16 \mathrm{~S}$ ribosomal RNA gene were amplified by PCR. The reaction parameters of PCR are as follows: $95^{\circ} \mathrm{C}$ for $5 \mathrm{~min}$, followed by 27 cycles at $95^{\circ} \mathrm{C}$ for $30 \mathrm{~s}, 55^{\circ} \mathrm{C}$ for $30 \mathrm{~s}$, and $72^{\circ} \mathrm{C}$ for $45 \mathrm{~s}$ and a final extension at $72^{\circ} \mathrm{C}$ for $10 \mathrm{~min}$. Specific primers are as follows. 341F 5'-barcode- CCTAYGGGRBGCASCAG-3' and 806R 5'- GGACTACNNGGGTATCTAAT-3'. PCR reactions were performed by TransStart Fastpfu DNA Polymerase (TransGen, China) using GeneAmp PCR System 9700 (ABI,U.S.). Amplicons were extracted from $2 \%$ agarose gels and purified using the AxyPrep DNA Gel Extraction Kit (Axygen Biosciences, Union City, CA, U.S.) according to the manufacturer's instructions. Amplicons were further quantified using QuantiFluor ${ }^{\mathrm{mt}}$-ST (Promega, U.S.). The pooled DNA product was used to construct Illumina Pair-End library following Illumina's genomic DNA library preparation procedure by using NEB NextUltra DNA Library Prep Kit for Illumina (NEB, USA). The library quality was assessed on the Qubit@2.0 Fluorometer (Thermo Scientific, CA, USA) and Agilent Bioanalyzer 2100 system (Agilent Technologies, CA, USA). Then the amplicon library was paired-end sequenced (2 $\times 250$ ) on an Illumina MiSeq platform (MingkeBio (Hangzhou) Co., Ltd., Hangzhou, China) according to the standard protocols.

\subsection{Bioinformatics analysis}

Paired-end reads generated on Illumina platform were carried out with QuantiFluor $^{\text {tm }}$-ST. Joined reads that have $\geq 97 \%$ proximity were assigned to OTUs. Prepare typical sequences of OTU and analyze the divergences of dominants. Alpha diversity was considered as Observed-species, Chaol, Shannon and Simpson. Beta diversity analysis was performed using UniFrac (Lozupone et al., 2011) to compare the results of the principal component analysis (PCA) with the community ecology package. $\mathrm{R}$-forge (Vegan 2.0 package was used to generate a PCA figure.
Table 1. The red meat diet in different groups.

\begin{tabular}{lcccc}
\hline Composition & $\begin{array}{c}\text { Control } \\
\text { group }\end{array}$ & $\begin{array}{c}\text { Low-dose } \\
\text { group }\end{array}$ & $\begin{array}{c}\text { Medium-dose } \\
\text { group }\end{array}$ & $\begin{array}{c}\text { High-dose } \\
\text { group }\end{array}$ \\
\hline tyrosine & 20 & 20 & 0 & 0 \\
red meat & 0 & 25 & 50 & 75 \\
corn starch & 41.72 & 16.7 & 11.7 & 2.19 \\
sucrose & 10.95 & 10.95 & 10.95 & 10.95 \\
sunflower seed & 17.66 & 17.68 & 17.68 & 2.19 \\
oil & & & & \\
lard & 2.55 & 2.55 & 2.55 & 2.55 \\
Alpha-cellulose & 2 & 2 & 2 & 2 \\
L-cysteine & 0.3 & 0.3 & 0.3 & 0.3 \\
choline & 0.17 & 0.17 & 0.17 & 0.17 \\
mineral & 3.5 & 3.5 & 3.5 & 3.5 \\
vitamin & 1 & 1 & 1 & 1 \\
DL-methionine & 0.15 & 0.15 & 0.15 & 0.15 \\
Total & 100 & 100 & 100 & 100 \\
\hline
\end{tabular}

\section{Sequence length distribution}

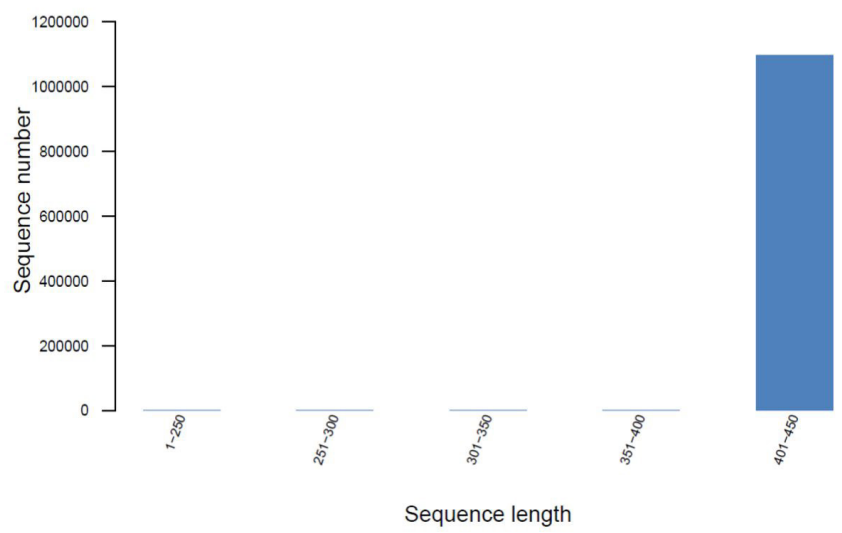

Figure 1. All the trimmed sequences length. $\mathrm{X}$-axis is the sequence length and $\mathrm{Y}$-axis is the sequence number.

Venn diagrams were implemented by Venn Diagram software, while Mantel test, Redundancy analysis (RDA) and Heatmap figures were performed by R package vegan.

\subsection{Statistical analysis}

The quality control and sequence filtering of amplicons were performed according to the barcode matching and sequence overlapping with QIIME. OTUs were clustered with 97\% similarity cutoff using Usearch (version 10) and chimeric sequences were identified and removed using UCHIME. The phylogenetic affiliation of each 16S rRNA gene sequence was analyzed by RDP Classifier against the silva (SSU123)16S rRNA database using confidence threshold of 70\% (Amato et al., 2013).

Community compositional was analyzed using linear discriminant analysis (LDA) and effect size (LEfSe) (Segata et al., 2011). Differences between groups were analyzed by one-way analysis of variance (ANOVA) test followed by Student's t-test. 
Data are presented as means \pm standard deviation $(\mathrm{SD}) . \mathrm{P}<0.05$ was considered significant. A P-value between 0.05 and 0.1 was considered a tendency. Statistical analysis was performed using SPSS 20 (International Business Machines, corp., Armonk, NY, USA).

\section{Results and discussion}

\subsection{Statistics of trimmed sequences}

Lengths of all the trimmed sequences were shown in Figure 1. The proportion of the high-quality sequence was more than $95 \%$. The sequence length was almost between 401 and $450 \mathrm{bp}$, with the average value of $414.15 \mathrm{bp}$.

\subsection{Rarefaction curves}

The species richness was evaluated as previous described (Schloss et al., 2011). As shown in Figure 2, the rarefaction curves of all the samples were common risen rapidly and then flattened. Only the 1-2 and 1-5 samples showed saturation. Similarly, they had the lower diversity and richness than others.

\subsection{Distribution and accuracy of statistical analyses}

As shown in Table 2, after filtering, entire 1,098,609 quality sequences from enrichment samples were obtained, with an average of $44520 \pm 9209$ reads per sample. There were 13960 OTUs with $97 \%$ similarity. The community diversity characteristics of the 4 reactors were also showed in Table 2. Chao index indicated the OTU richness The Chao, Shannon and Simpson indices suggested that the species diversity in the control group was decreased than red meat diet groups, and those in both medium-dose group and high-dose group were higher than other groups (Table 3). Samples in the control group can decrease the growth of specific microorganisms due to the lower OTU numbers $(465 \pm 28)$. Although most of the intestinal microbiota is the dominant flora, there are still harmful ones. Along with the increased proportion of red meat in diet, the number of dominant flora was decreased while the harmful

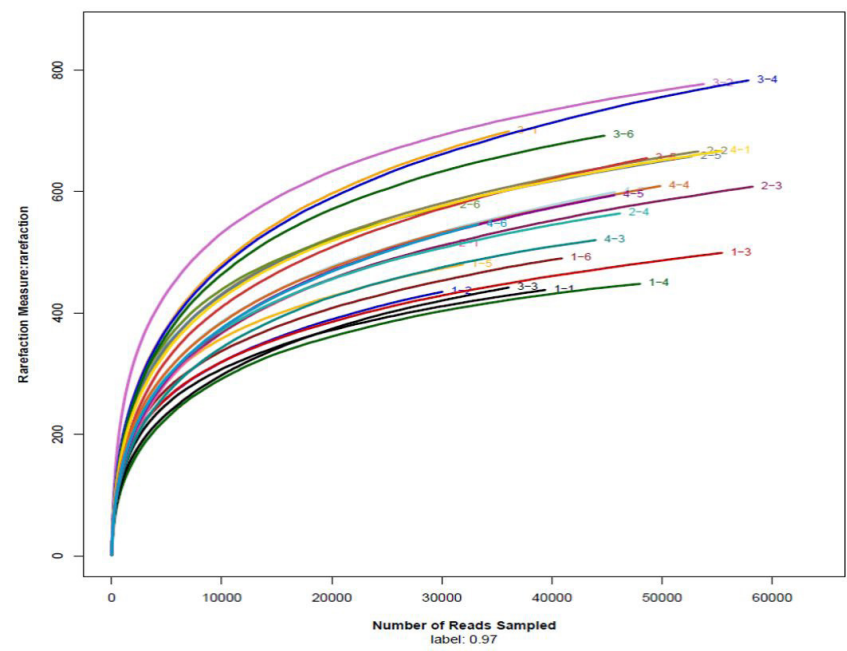

Figure 2. The rarefaction curves of all the samples. $X$-axis is the number of readers sampled and Y-axis is rarefaction measure. Different colors represent mice in different groups. flora was increased, which can lead to an elevated a-diversity of the intestinal flora.

\subsection{Shannon-Wiener and rank-abundance curve analysis}

Shannon-Wiener index is taken into account species richness and proportion of each species (Magwira et al., 2012). As shown in Figure 3a, the Shannon-Wiener curve of all samples monotonically climbed until reaching a plateau. Figure $3 \mathrm{~b}$ showed the rank-abundance curves. The width reflected the abundance.

\subsection{Venn diagram and principal coordinate analysis}

There were 948, 1072, 1123 and 1030 OTUs in the control group, low-dose group, medium-dose group and high-dose group, respectively (Figure 4). Among the four groups, there were 603 common OTUs. 77 unique OTUs were found in the control group, which was the largest in the four groups. This could be a hint of the beneficial effect of diet.
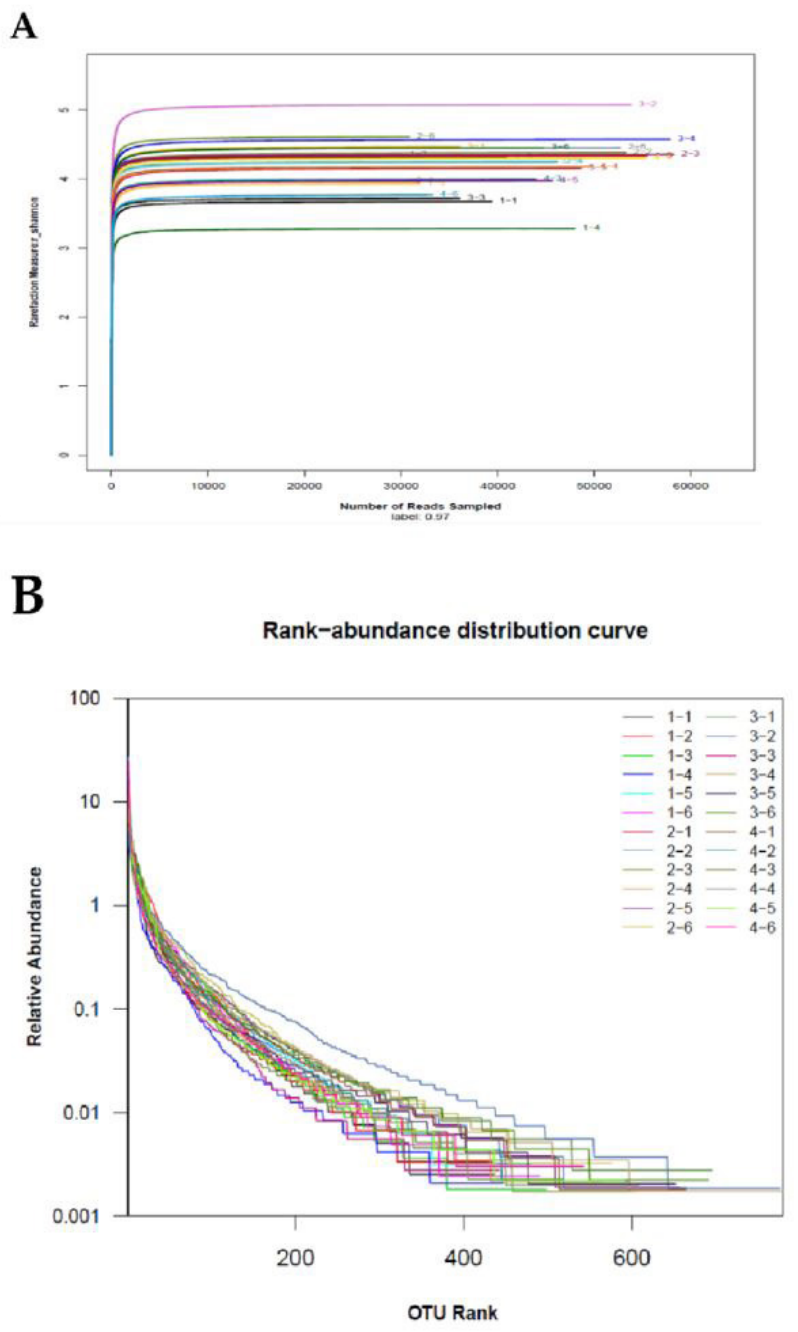

Figure 3. Shannon-Wiener and rank-abundance curve analysis. (a) the Shannon-Wiener curve of all samples; $\mathrm{X}$-axis is the number of readers sampled and $\mathrm{Y}$-axis is rarefaction measure. (b) the rank-abundance curves of all samples. X-axis is the OUT Rank and Y-axis is relative abundance. The width of the curve reflects the species richness. Different colors represent mice in different groups. 
Original Article

Effects of red meat diet on gut microbiota in mice

Table 2. The community diversity characteristics of OTUs.

\begin{tabular}{|c|c|c|c|c|c|c|}
\hline \multirow{2}{*}{ Sample ID } & \multirow{2}{*}{ Reads } & \multicolumn{5}{|c|}{0.97} \\
\hline & & OTU & Chao & Coverage & Shannon & Simpson \\
\hline $1-1$ & & & $-515,652$ & & $(3.66,3.69)$ & $(0.0661,0.0687)$ \\
\hline $1-2$ & & & $-516,647$ & & $(4.34,4.37)$ & $(0.0249,0.026)$ \\
\hline \multirow[t]{2}{*}{$1-3$} & 55429 & 499 & 634 & 0.997835 & 4.34 & 0.0255 \\
\hline & & & $-583,715$ & & $(4.32,4.35)$ & $(0.0251,0.0259)$ \\
\hline $1-4$ & & & $-484,552$ & & $(3.26,3.3)$ & $(0.11,0.1138)$ \\
\hline \multirow[t]{2}{*}{$1-5$} & 31901 & 480 & 641 & 0.996301 & 3.93 & 0.0828 \\
\hline & & & $-579,741$ & & $(3.91,3.95)$ & $(0.0803,0.0852)$ \\
\hline \multirow[t]{2}{*}{$1-6$} & 40893 & 490 & 613 & 0.997066 & 4.32 & 0.0274 \\
\hline & & & $-567,687$ & & $(4.3,4.33)$ & $(0.0269,0.0278)$ \\
\hline $2-1$ & 30734 & 514 & 640 & 0.99564 & 3.98 & 0.0468 \\
\hline \multirow[t]{2}{*}{$2-3$} & 58219 & 608 & 768 & 0.997406 & 4.36 & 0.0246 \\
\hline & & & $-713,851$ & & $(4.34,4.37)$ & $(0.0243,0.025)$ \\
\hline \multirow[t]{2}{*}{$2-4$} & 46163 & 564 & 706 & 0.997119 & 4.25 & 0.0302 \\
\hline & & & $-655,785$ & & $(4.24,4.26)$ & $(0.0297,0.0307)$ \\
\hline \multirow[t]{2}{*}{$2-5$} & 52696 & 658 & 838 & 0.997153 & 4.45 & 0.0353 \\
\hline & & & $-776,934$ & & $(4.44,4.47)$ & $(0.0345,0.0361)$ \\
\hline \multirow[t]{2}{*}{$2-6$} & 30820 & 577 & 698 & 0.996009 & 4.61 & 0.0242 \\
\hline & & & $-653,769$ & & $(4.59,4.63)$ & $(0.0236,0.0248)$ \\
\hline \multirow[t]{2}{*}{$3-1$} & 36069 & 699 & 849 & 0.995259 & 4.47 & 0.0328 \\
\hline & & & $-800,920$ & & $(4.45,4.48)$ & $(0.032,0.0336)$ \\
\hline $3-2$ & 53789 & 777 & 879 & 0.99749 & 5.08 & 0.013 \\
\hline $3-6$ & & & $-766,864$ & & $(4.44,4.47)$ & $(0.025,0.0258)$ \\
\hline $4-1$ & 55401 & 667 & 854 & 0.99722 & 4.3 & 0.0349 \\
\hline & & & $-790,952$ & & $(4.29,4.32)$ & $(0.0343,0.0356)$ \\
\hline $4-2$ & 45717 & 599 & 762 & 0.99661 & 4.23 & 0.0307 \\
\hline & & & $-707,846$ & & $(4.22,4.25)$ & $(0.0302,0.0311)$ \\
\hline $4-3$ & 43952 & 520 & 621 & 0.997361 & 4 & 0.0442 \\
\hline & & & $-583,682$ & & $(3.98,4.01)$ & $(0.0434,0.045)$ \\
\hline $4-4$ & 49831 & 609 & 811 & 0.996789 & 4.18 & 0.0374 \\
\hline & & & $-742,915$ & & $(4.16,4.19)$ & $(0.0368,0.0381)$ \\
\hline $4-5$ & 45657 & 594 & 764 & 0.996539 & 3.98 & 0.051 \\
\hline & & & $-707,850$ & & $(3.96,3.99)$ & $(0.0499,0.0521)$ \\
\hline $4-6$ & 33257 & 546 & 732 & 0.995309 & 3.77 & 0.0819 \\
\hline & & & $-669,828$ & & $(3.75,3.79)$ & $(0.0797,0.084)$ \\
\hline
\end{tabular}

OTU, operational taxonomic unit; ID, identification.

\subsection{Community structure analysis}

Table 4 showed that there were 21 phyla, 42 classes, 92 orders, 140 families, 268 genera and 335 species in the communities of all samples. The dominant phyla of all groups were Firmicutes,
Bacteroidetes, Proteobacteria and Actinobacteria, while the dominant classes of all groups were Clostridia, Bacteroidia, Deltaproteobacteria and Bacilli. At the phylum level, the most predominant phylum was Firmicutes that contributed 59.33\%, 
Table 3. Alpha diversity index of fecal samples of mice in each group.

\begin{tabular}{clcrr}
\hline Groups & Chao index & Coverage index & Shannon index & \multicolumn{1}{c}{ Simpson index } \\
\hline Control & $588.17 \pm 50.515$ & $0.997150 \pm 0.000799$ & $3.9817 \pm 0.44061$ & $0.056733 \pm 0.036481$ \\
Low-dose & $742.83 \pm 74.497^{*}$ & $0.996758 \pm 0.000739$ & $4.3383 \pm 0.21198$ & $0.0322 \pm 0.008349$ \\
Medium-dose & $817.67 \pm 149.414^{* *}$ & $0.996585 \pm 0.000749$ & $4.4083 \pm 0.45314$ & $0.033083 \pm 0.016509$ \\
High-dose & $757.33 \pm 79.397^{* *}$ & $0.996638 \pm 0.000729$ & $4.0767 \pm 0.19664$ & $0.046683 \pm 0.018684$ \\
\hline
\end{tabular}

${ }^{\star} \mathrm{P}<0.05 ;{ }^{*} \mathrm{P}<0.01$ compared with the control group.

Table 4. The community structures of the observed samples at phylum levels.

\begin{tabular}{lcccc}
\hline \multicolumn{1}{c}{ Phylum } & Control group & Low-dose group & Medium-dose group & High-dose group \\
\hline Firmicutes & $59.33 \pm 15.95$ & $58.04 \pm 15.4$ & $52.58 \pm 13.69$ & $45.58 \pm 8.72$ \\
Bacteroidetes & $25.41 \pm 18.01$ & $26.87 \pm 20.75$ & $32.35 \pm 12.98$ & $39.85 \pm 13.53$ \\
Proteobacteria & $12.3 \pm 15.1$ & $9.82 \pm 7.92$ & $10.67 \pm 5.66$ & $7.69 \pm 3.89$ \\
Actinobacteria & $0.85 \pm 0.68$ & $2.98 \pm 1.77^{*}$ & $1.95 \pm 2.11$ & $1.35 \pm 2.09$ \\
Patescibacteria & $0.58 \pm 0.69$ & $1.35 \pm 0.53$ & $1.77 \pm 1.49^{*}$ & $1.56 \pm 0.79$ \\
Verrucomicrobia & $0.78 \pm 1.28$ & $0 \pm 0$ & $0.13 \pm 0.17$ & $0.63 \pm 1.42$ \\
Epsilonbacteraeota & $0.38 \pm 0.28$ & $0.16 \pm 0.18$ & $0.2 \pm 0.2$ & $2.52 \pm 4.42$ \\
Deferribacteres & $0.22 \pm 0.22$ & $0.01 \pm 0.01^{*}$ & $0.05 \pm 0.07$ & $0.16 \pm 0.24$ \\
Tenericutes & $0.11 \pm 0.11$ & $0.33 \pm 0.35$ & $0.18 \pm 0.17$ & $0.6 \pm 0.62$ \\
Cyanobacteria & $0.02 \pm 0.03$ & $0.39 \pm 0.79$ & $0.04 \pm 0.08$ & $0.03 \pm 0.05$ \\
Acidobacteria & $0.01 \pm 0.01$ & $0.01 \pm 0.01$ & $0.03 \pm 0.02$ & $0.01 \pm 0$ \\
Chloroflexi & $0 \pm 0$ & $0 \pm 0$ & $0.01 \pm 0.01$ & $0.01 \pm 0$ \\
Gemmatimonadetes & $0 \pm 0.01$ & $0 \pm 0$ & $0.02 \pm 0.01^{*}$ & $0 \pm 0$ \\
Nitrospirae & $0 \pm 0$ & $0.01 \pm 0.01$ & $0.01 \pm 0.01$ & $0 \pm 0.01$ \\
Unclassified & $0 \pm 0$ & $0.01 \pm 0.02$ & & $0.01 \pm 0.02$ \\
\hline
\end{tabular}

${ }^{*} \mathrm{P}<0.05$ compared with the control group.

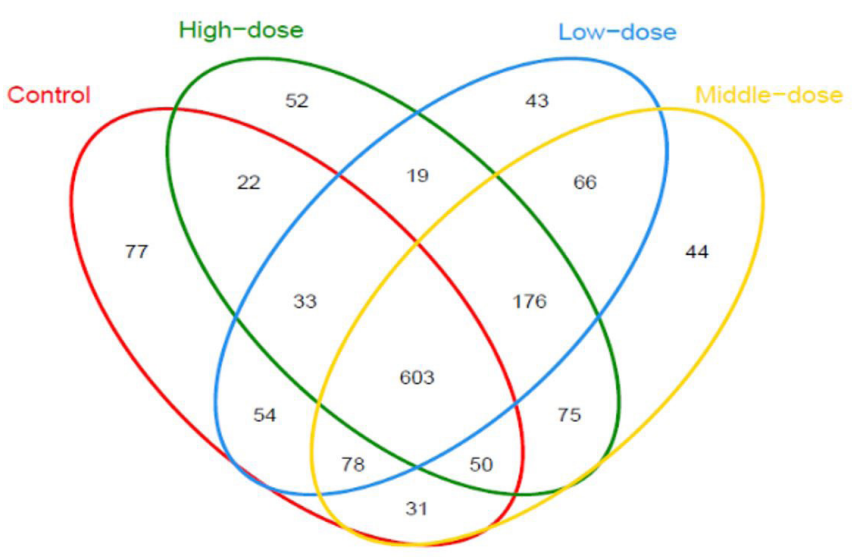

Figure 4. The Venn diagram. Red represents control group; green represents high-dose group; blue represents low-dose group and yellow represents middle-dose group.

$58.04 \%, 52.58 \%$ and $45.58 \%$ of the fecal microbiota in the control, low-dose, medium-dose and high-dose groups, respectively. The second predominant phylum was Bacteroidetes, contributing $25.4 \%, 26.87 \%, 32.35 \%$ and $39.85 \%$, respectively. There were increased proportion of Bacteroidetes (by 6.95\% and 14.44\%, respectively) in both medium-dose and high-dose groups. However, the proportion of Firmicutes was substantially reduced in these two groups (by $6.75 \%$ and $13.74 \%$, respectively). The results were shown in Figure 5.
As shown in Table 5, it demonstrated that the dominant family of all groups were Lachnospiraceae, Ruminococcaceae, Muribaculaceae, Desulfovibrionaceae. Lachnospiraceae, Ruminococcaceae, Muribaculaceae, Burkholderiaceae, Erysipelotrichaceae, Lactobacillaceae, Marinifilaceae, Caulobacteraceae and Peptococcaceae were lower in the highdose group than those in the control group. Clostridiaceae 1, Enterococcaceae and Peptostreptococcaceae were significantly lower in the medium-dose and high-dose group than those in the control group $(\mathrm{P}<0.01)$. Two family were notably higher in high-dose group than control group, including Bacteroidaceae ( $\mathrm{P}$ $<0.01)$ and Family XIII $(\mathrm{P}<0.01)$. Moreover, Desulfovibrionaceae, Rikenellaceae, Prevotellaceae and Helicobacteraceae were higher in the high-dose group than those in the control group. At genera levels, Parasutterella, Marvinbryantia, [Eubacterium] nodatum group, Bacteroides, Lachnospiraceae UCG-001, Adlercreutzia, Prevotellaceae UCG-001, Ruminococcaceae UCG013, Negativibacillus, Faecalitalea, Lachnospiraceae UCG-008 and [Eubacterium] brachy group were higher in the high-dose group than those in the control group $(\mathrm{P}<0.05)$ (Figures 6-8).

The gastrointestinal tracts in humans and other animals harbor a diverse array of microorganisms, which play fundamental-roles in health and disease. Imbalance in the gut microbiota, namely dysbiosis, can lead to various diseases, ranging from metabolic and cardiovascular disorders to cancer and gastrointestinal tract disorders. Approaches to improve gut dysbiosis, including dietary intervention, intake of probiotics and fecal microbiota transplantation, are emerging strategies to 


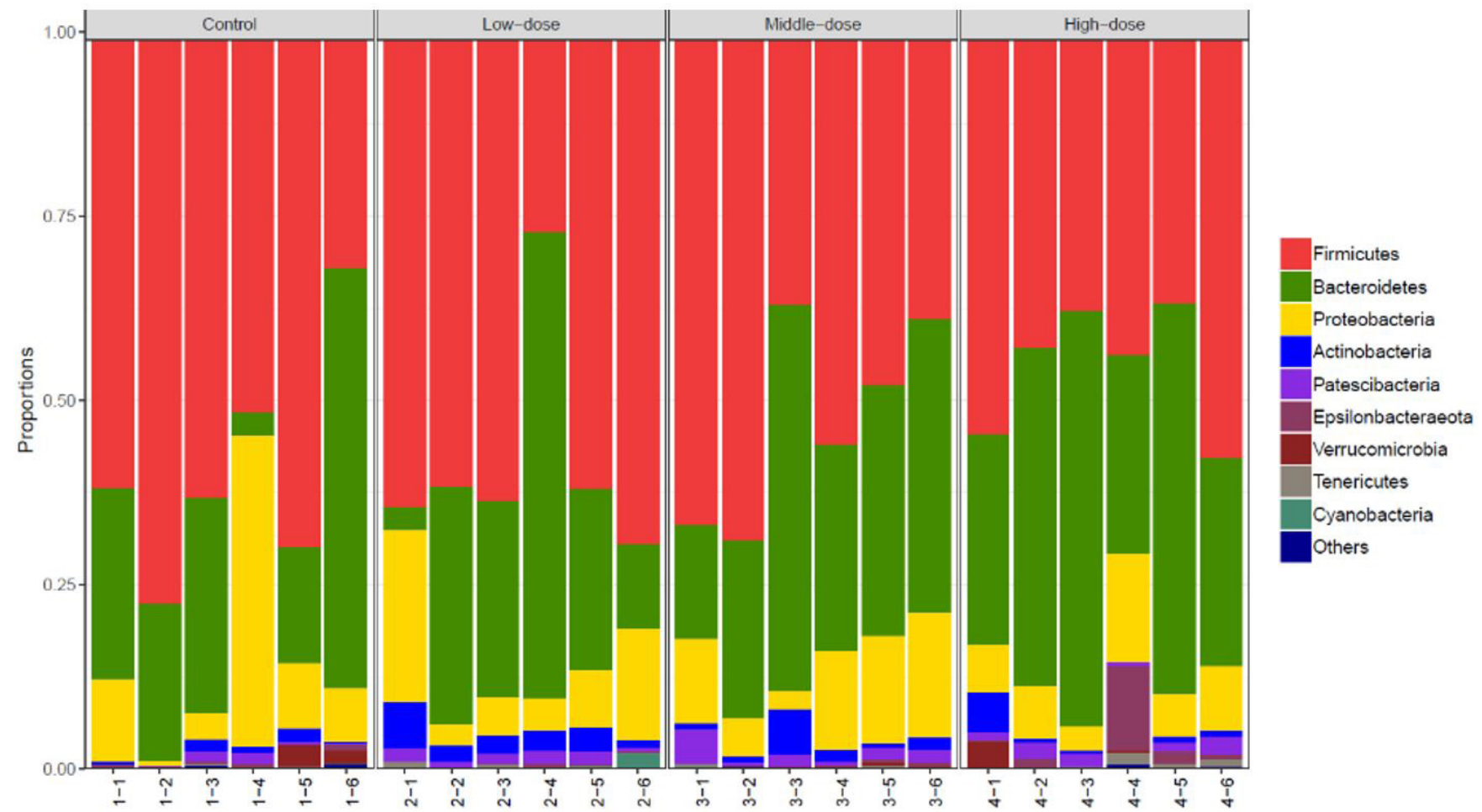

Figure 5. The community structures at phylum levels. X-axis is the mice in different groups and Y-axis is proportions. Different colors represent different bacteria.

Table 5. The community structures of the observed samples at family levels.

\begin{tabular}{|c|c|c|c|c|}
\hline Family & Control group & Low-dose group & Middle-dose group & High-dose group \\
\hline Lachnospiraceae & $34.1 \pm 10.93$ & $36.9 \pm 14.16$ & $32.84 \pm 13.53$ & $31.39 \pm 9.22$ \\
\hline Ruminococcaceae & $15.57 \pm 13.25$ & $12.97 \pm 3.54$ & $13.32 \pm 6.61$ & $9.83 \pm 4.42$ \\
\hline Muribaculaceae & $14 \pm 8.9$ & $19.78 \pm 15.41$ & $23.03 \pm 13.78$ & $11.62 \pm 6.71$ \\
\hline Desulfovibrionaceae & $6.56 \pm 4.1$ & $5.9 \pm 4.57$ & $8.64 \pm 4.83$ & $6.84 \pm 3.61$ \\
\hline Rikenellaceae & $5.02 \pm 4.54$ & $3.18 \pm 2.82$ & $2.7 \pm 1.39$ & $8.69 \pm 3.24$ \\
\hline Burkholderiaceae & $4.73 \pm 11.56$ & $0.17 \pm 0.17$ & $1.69 \pm 2.3$ & $0.64 \pm 0.55$ \\
\hline Erysipelotrichaceae & $2.54 \pm 2.22$ & $2.33 \pm 1.8$ & $0.36 \pm 0.41$ & $0.13 \pm 0.1$ \\
\hline Clostridiaceae 1 & $2.41 \pm 5.6$ & $0.01 \pm 0.01^{\star}$ & $0 \pm 0^{\star \star}$ & $0 \pm 0^{\star \star}$ \\
\hline Lactobacillaceae & $2.18 \pm 2.48$ & $3.86 \pm 3.68$ & $4.74 \pm 6.25$ & $1.01 \pm 1.11$ \\
\hline Prevotellaceae & $2.11 \pm 2.85$ & $0.2 \pm 0.23$ & $0.35 \pm 0.53$ & $2.57 \pm 3.7$ \\
\hline Marinifilaceae & $2.11 \pm 2.13$ & $1.19 \pm 1.36$ & $0.83 \pm 1.04$ & $0.29 \pm 0.1$ \\
\hline Tannerellaceae & $1.4 \pm 2.22$ & $0.39 \pm 0.63$ & $0.6 \pm 0.54$ & $1.85 \pm 1.17$ \\
\hline Bacteroidaceae & $0.73 \pm 0.88$ & $2.11 \pm 1.87$ & $4.83 \pm 6.15$ & $14.82 \pm 6.77^{\star \star}$ \\
\hline Family XIII & $0.23 \pm 0.22$ & $0.57 \pm 0.35$ & $0.67 \pm 0.33$ & $2.11 \pm 1.12^{\star \star}$ \\
\hline Caulobacteraceae & $0.21 \pm 0.34$ & $1.97 \pm 1.85^{\star}$ & $0.11 \pm 0.16$ & $0.07 \pm 0.06$ \\
\hline Eggerthellaceae & $0.73 \pm 0.69$ & $2.84 \pm 1.64^{\star}$ & $1.88 \pm 2.06$ & $1.33 \pm 2.07$ \\
\hline Enterococcaceae & $0.12 \pm 0.16$ & $0 \pm 0$ & $0 \pm 0^{* *}$ & $0 \pm 0.01$ \\
\hline Helicobacteraceae & $0.38 \pm 0.28$ & $0.16 \pm 0.18$ & $0.2 \pm 0.2$ & $2.51 \pm 4.42$ \\
\hline Mollicutes RF39_norank & $0.05 \pm 0.07$ & $0.14 \pm 0.12$ & $0.1 \pm 0.15$ & $0.11 \pm 0.12$ \\
\hline Peptococcaceae & $0.35 \pm 0.24$ & $0.21 \pm 0.23$ & $0.34 \pm 0.34$ & $0.13 \pm 0.05$ \\
\hline Peptostreptococcaceae & $0.91 \pm 1.42$ & $0.75 \pm 1.01$ & $0.05 \pm 0.07^{*}$ & $0 \pm 0^{\star *}$ \\
\hline
\end{tabular}

${ }^{*} \mathrm{P}<0.05 ;{ }^{* *} \mathrm{P}<0.01$ compared with the control group.

treat diseases (Cheung et al., 2020). Diet can significantly reshape gut microbial composition (Brown et al., 2012). For instance, compared to European children, increased Bacteroidetes and decreased Firmicutes and Enterobacteriaceae in rural African children were mainly attributed to differences in dietary patterns between the two populations. This difference was probably 


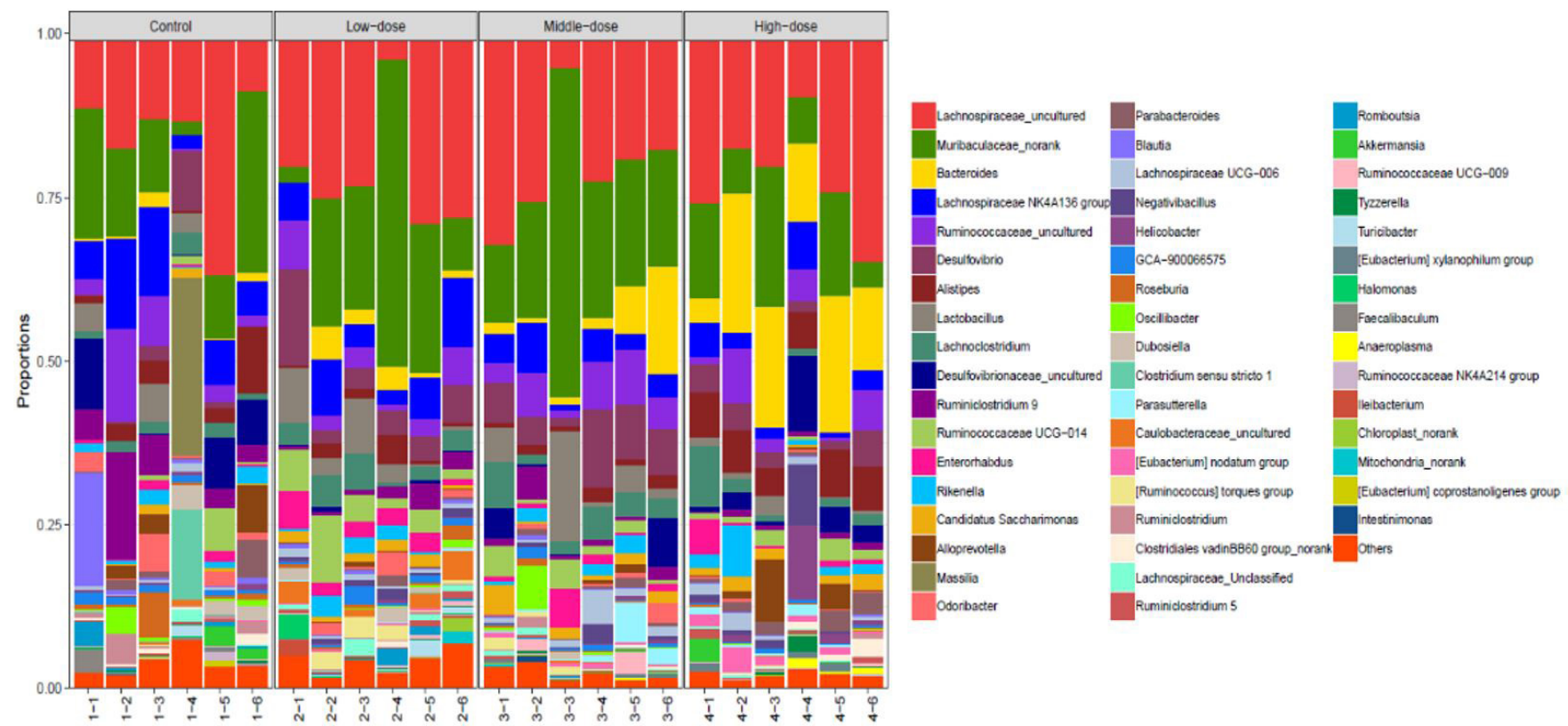

Figure 6. The community structures at genera levels. X-axis is the mice in different groups and Y-axis is proportions. Different colors represent different bacteria.

\section{Cladogram}
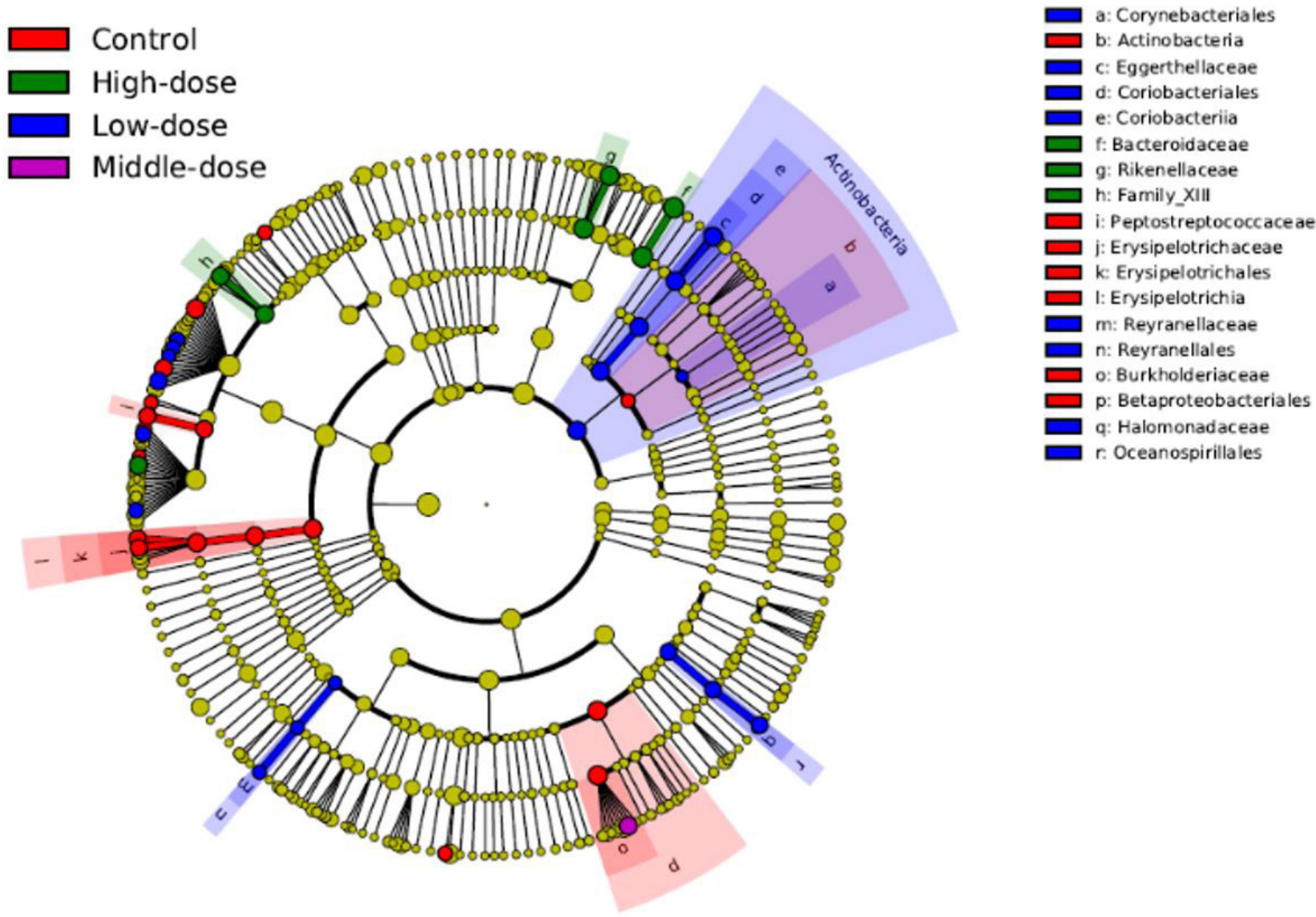

Figure 7. Significantly different species were compared in the four groups. 


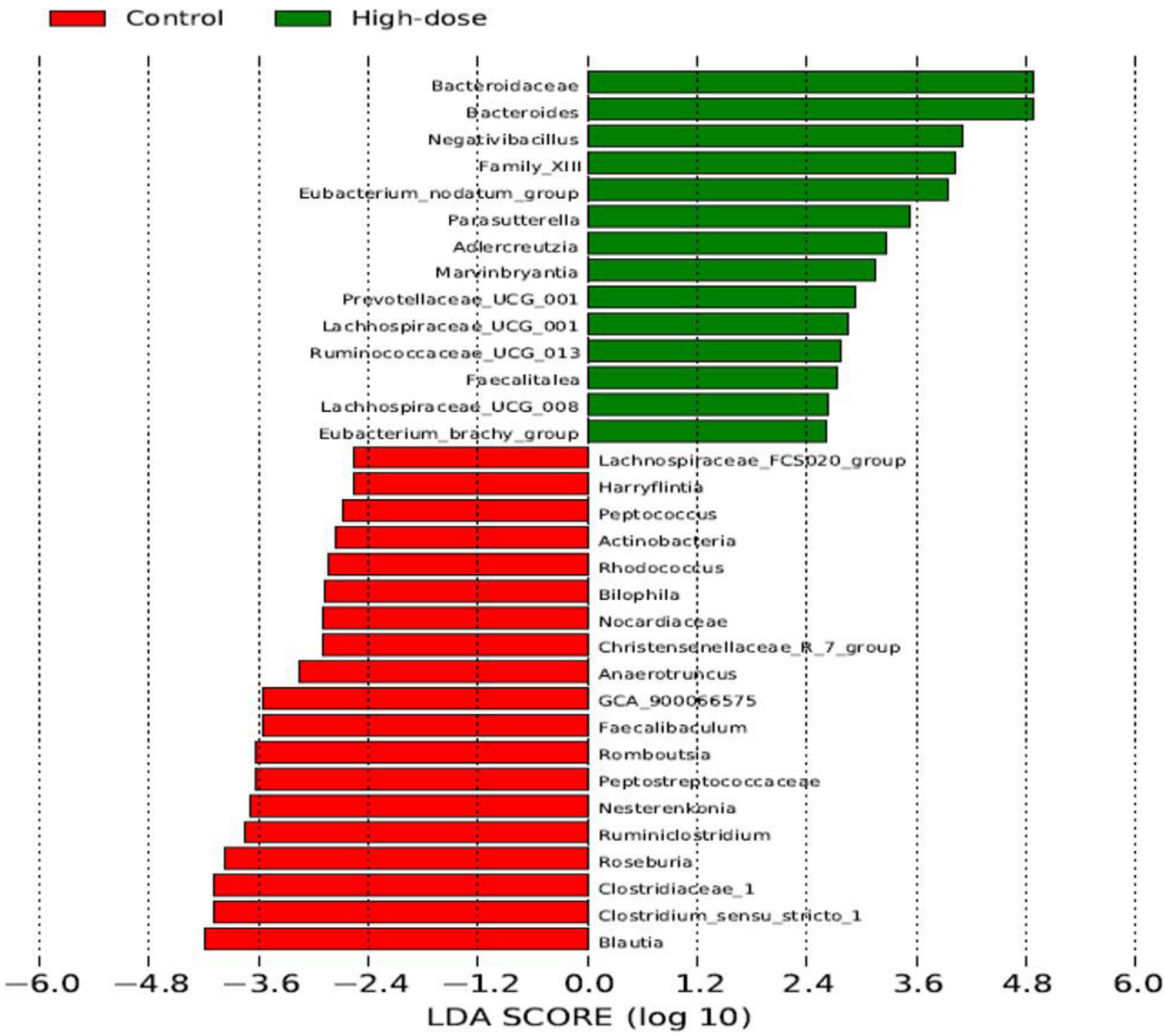

Figure 8. Significantly different species were compared in the high-dose group than in the control group.

explained by the higher red meat content of the food in Europe (De Filippo et al., 2010). In this study, at the phylum level, there were four major phyla (Firmicutes, Bacteroidetes, Proteobacteria and Actinobacteria) in all samples. We found that Firmicutes and Bacteroides were the most abundant flora in mice' intestines. Bacteroidetes and Firmicutes are leading groups of bacteria involved in metabolizing undigested food (Parkar et al., 2013). Moreover, the abundance of Bacteroidetes in the high-dose group was higher than that in the control group (39.85\% vs. $25.40 \%$ ), while the abundance of Firmicutes in the high-dose group was lower than that in the control group ( $49.58 \%$ vs. $59.33 \%$ ). This is consistent with those researches on humans. Both short- and long-term studies have shown a higher proportion of Bacteroides in fecal microbiota after the consumption of a "Western diet" rich in animal protein and fat, and low in fibre (Wu et al., 2011; David et al., 2014).

Compared to omnivores, vegetarians have significantly lower microbial counts of Bacteroides and Bifidobacterium specie, while the total cell numbers remain unchanged (Zimmer et al., 2012). In this study, the contents of Bacteroidaceae and Family XIII were significantly higher in the high-dose group than the control group. The abundance of Lactobacillus was low in all groups, contributing $2.18 \%, 3.86 \%, 4.74 \%$ and $1.01 \%$, respectively. It's noteworthy that the lowest occurred in the high-dose group. Lactobacillus has been considered as a key player in host metabolic balance (Arora et al., 2012). It is a common probiotic that can inhibit pathogens by fermenting food to produce lactic acid, which lowers the $\mathrm{pH}$ value in the intestine environment. 
Moreover, Lachnospiraceae is a highly abundant bacterial family within the gut microbiota in human, whose members may protect against human colon cancer by producing butyric acid, a substance that is important for both microbial and host epithelial cell growth (Meehan \& Beiko, 2014; Yang et al., 2020; Huang et al., 2020; Hwang et al., 2019; Grom et al., 2020). In this study, the compositions of Lachnospiraceae in the control, lowdose, medium-dose and high-dose groups were $34.1 \%$, 36.9\%, $32.84 \%$ and $31.39 \%$, respectively. Lachnospiraceae FCS020 group, Lachnospiraceae UCG-001 and Lachnospiraceae UCG-008 were significantly higher in the low-dose group than other groups. These results indicated that the red meat diet may reform the gut microbiota, and refinement of diet regarding the proportion of red meat could help prevent colon cancer.

On the genus level, there were several species with widely varying abundance in community heatmap, including Romboutsia, Roseburia, Lachnospiraceae FCS020 group, Clostridium sensu stricto 1, Anaerotruncus, Bilophila, Blautia, GCA-900066575, Christensenellaceae R-7 group, Nesterenkonia, Harryflintia, Peptococcus, Rhodococcus, Faecalibaculum, and Ruminiclostridium. They were all dominant bacterium in the control group. Mouse models revealed that Faecalibacterium prausnitzii had anti-inflammatory properties, and in patients with Crohn Disease, reductions in ileal F. prausnitzii abundance were associated with disease recurrence (Sokol et al., 2008). In patients with ulcerative disease (UC), from stool samples, reductions in F. prausnitzii abundance are associated with an increased number of disease flares, a shorter time in remission, and a higher disease extent (pancolitis versus proctitis) in adult UC patients (Varela et al., 2013). In our experiment, the abundance of the Faecalibaculum was the highest in the control group, and was gradually decreased with the increase of red meat diet. Therefore, red meat diet may alter intestinal flora and, as a result, increase the risk of inflammatory bowel disease by altering intestinal flora.

Our experiment also demonstrated that the red meat diet was associated with significant changes in the composition of intestinal flora at genus level in mice, including increased relative abundance of Bacteroidaceae and Rikenellaceae, and decreased ratio of Peptostreptococcaceae and Blautia and Erysipelotrichaceae. We found a higher abundance of the genus Roseburia in the control group, which was consistent with previously reported studies (Chen et al., 2014; Morgan et al., 2012; Rehman et al., 2016). The genus Roseburia includes known butyrate producing organisms, and butyrate can interact with the intestinal epithelium to produce an anti-inflammatory environment (Louis et al., 2014) and modulate intestinal barrier function (Kelly et al., 2015). In patients with UC, a decrease in short-chain fatty acid production is correlated with reductions in Roseburia abundance (Machiels et al., 2014; Kumari et al., 2013). Therefore, the loss of Roseburia may lead to the uncontrolled colonic inflammation of UC. In general, the red meat diet changes the intestinal flora, which causes a decrease in Roseburia. This pathway may explain why people are prone to ulcerative colitis prefer red meat.

The mice in the control group had a higher abundance of Clostridiaceae-1, Clostridium spp, which contributed to complex carbohydrate breakdown in the gut and produces short chain acids. It is beneficial to intestinal epithelial cells (Kaoutari et al., 2013). The Lachnospiraceae and Ruminococcaceae are two of the most abundant families from the order Clostridiales found in the mammalian gut environment, and they have been demonstrated to be associated with the maintenance of gut health (Zhang et al., 2018). In this study, we found that Clostridiaceae-1 and Ruminococcaceae were significantly higher in the control and low-dose group compared with other groups. It further confirmed our hypothesis that less red meat intake might have a healthier gut flora.

\section{Conclusions}

Remarkably, the profiling of the gut microbiota communities with various diets in this investigation showed that administration of red meat diet for 8 weeks caused a reshaping of the intestinal bacteria composition towards an unhealthier microbial community. The red meat diet changed fecal microbiota content significantly by enriching harmful bacteria and/or depleting the beneficial ones. Therefore, it is necessary to continue the evaluation of the diet as a modulator of the gut microbiota, which could provide potential treatment strategies for diseases such as colitis and cancers.

\section{Funding}

This research was funded by the National Natural Science Foundation of China (grant number: 81760100).

\section{References}

Abu-Ghazaleh, N., Chua, W. J., \& Gopalan, V. (2021). Intestinal microbiota and its association with colon cancer and red/processed meat consumption. Journal of Gastroenterology and Hepatology, 36(1), 75-88. http://dx.doi.org/10.1111/jgh.15042. PMid:32198788.

Alexander, D. D., \& Cushing, C. A. (2011). Red meat and colorectal cancer: a critical summary of prospective epidemiologic studies. Obesity Reviews, 12(5), e472-e493. http://dx.doi.org/10.1111/j.1467789X.2010.00785.x. PMid:20663065.

Amato, K. R., Yeoman, C. J., Kent, A., Righini, N., Carbonero, F., Estrada, A., Rex Gaskins, H., Stumpf, R. M., Yildirim, S., Torralba, M., Gillis, M., Wilson, B. A., Nelson, K. E., White, B. A., \& Leigh, S. R. (2013). Habitat degradation impacts black howler monkey (Alouatta pigra) gastrointestinal microbiomes. The ISME Journal, 7(7), 1344-1353. http://dx.doi.org/10.1038/ismej.2013.16. PMid:23486247.

Arora, T., Anastasovska, J., Gibson, G., Tuohy, K., Sharma, R. K., Bell, J., \& Frost, G. (2012). Effect of Lactobacillus acidophilus NCDC 13 supplementation on the progression of obesity in diet-induced obese mice. The British Journal of Nutrition, 108(8), 1382-1389. http://dx.doi.org/10.1017/S0007114511006957. PMid:22289627.

Birt, D. F., \& Phillips, G. J. (2014). Diet, genes, and microbes: complexities of colon cancer prevention. Toxicologic Pathology, 42(1), 182-188. http://dx.doi.org/10.1177/0192623313506791. PMid:24129759.

Brown, K., DeCoffe, D., Molcan, E., \& Gibson, D. L. (2012). Diet-induced dysbiosis of the intestinal microbiota and the effects on immunity and disease. Nutrients, 4(8), 1095-1119. http://dx.doi.org/10.3390/ nu4081095. PMid:23016134.

Butel, M. J., Waligora-Dupriet, A. J., \& Wydau-Dematteis, S. (2018). The developing gut microbiota and its consequences for health. Journal of Developmental Origins of Health and Disease, 9(6), 590597. http://dx.doi.org/10.1017/S2040174418000119. PMid:29562949. 
Chen, L., Wang, W., Zhou, R., Ng, S. C., Li, J., Huang, M., Zhou, F., Wang, X., Shen, B., A Kamm, M., Wu, K., \& Xia, B. (2014). Characteristics of fecal and mucosa-associated microbiota in Chinese patients with inflammatory bowel disease. Medicine, 93(8), e51. http://dx.doi. org/10.1097/MD.0000000000000051. PMid:25121355.

Cheung, M. K., Yue, G. G. L., Chiu, P. W. Y., \& Lau, C. B. S. (2020). A review of the effects of natural compounds, medicinal plants, and mushrooms on the gut microbiota in colitis and cancer. Frontiers in Pharmacology, 11, 744. http://dx.doi.org/10.3389/fphar.2020.00744. PMid:32499711.

David, L. A., Maurice, C. F., Carmody, R. N., Gootenberg, D. B., Button, J. E., Wolfe, B. E., Ling, A. V., Devlin, A. S., Varma, Y., Fischbach, M. A., Biddinger, S. B., Dutton, R. J., \& Turnbaugh, P. J. (2014). Diet rapidly and reproducibly alters the human gut microbiome. Nature, 505(7484), 559-563. http://dx.doi.org/10.1038/nature12820. PMid:24336217.

De Filippo, C., Cavalieri, D., Di Paola, M., Ramazzotti, M., Poullet, J. B., Massart, S., Collini, S., Pieraccini, G., \& Lionetti, P. (2010). Impact of diet in shaping gut microbiota revealed by a comparative study in children from Europe and rural Africa. Proceedings of the National Academy of Sciences of the United States of America, 107(33), 1469114696. http://dx.doi.org/10.1073/pnas.1005963107. PMid:20679230.

Dey, P. (2019). Gut microbiota in phytopharmacology: A comprehensive overview of concepts, reciprocal interactions, biotransformations and mode of actions. Pharmacological Research, 147, 104367. http:// dx.doi.org/10.1016/j.phrs.2019.104367. PMid:31344423.

Grom, L. C., Rocha, R. S., Balthazar, C. F., Guimarães, J. T., Coutinho, N. M., Barros, C. P., Pimentel, T. C., Venâncio, E. L., Collopy, I. Jr., Maciel, P. M. C., Silva, P. H. F., Granato, D., Freitas, M. Q., Esmerino, E. A., Silva, M. C., \& Cruz, A. G. (2020). Postprandial glycemia in healthy subjects: Which probiotic dairy food is more adequate? Journal of Dairy Science, 103(2), 1110-1119. http://dx.doi.org/10.3168/ jds.2019-17401. PMid:31785881.

Hudcovic, T., Stěpánková, R., Cebra, J., \& Tlaskalová-Hogenová, H. (2001). The role of microflora in the development of intestinal inflammation: acute and chronic colitis induced by dextran sulfate in germ-free and conventionally reared immunocompetent and immunodeficient mice. Folia Microbiologica, 46(6), 565-572. http:// dx.doi.org/10.1007/BF02818004. PMid:11898350.

Hwang, J. S., Lee, S. B., Choi, M.-J., Kim, J. T., \& Seo, H. G. (2019). Anti-adipogenic effect of a turmeric extract-loaded nanoemulsion in 3T3-L1 preadipocytes and high fat diet-fed mice. Food Science and Technology (Campinas), 39(Suppl. 2), 439-447. http://dx.doi. org/10.1590/fst.20718.

Ijssennagger, N., Belzer, C., Hooiveld, G. J., Dekker, J., van Mil, S. W., Müller, M., Kleerebezem, M., \& van der Meer, R. (2015). Gut microbiota facilitates dietary heme-induced epithelial hyperproliferation by opening the mucus barrier in colon. Proceedings of the National Academy of Sciences of the United States of America, 112(32), 1003810043. http://dx.doi.org/10.1073/pnas.1507645112. PMid:26216954.

Jiang, L., \& Schnabl, B. (2020). Gut Microbiota in Liver Disease: What Do We Know and What Do We Not Know? Physiology (Bethesda, MD), 35(4), 261-274. http://dx.doi.org/10.1152/physiol.00005.2020. PMid:32490750.

Kaoutari, A. E., Armougom, F., Gordon, J. I., Raoult, D., \& Henrissat, B. (2013). The abundance and variety of carbohydrate-active enzymes in the human gut microbiota. Nature Reviews. Microbiology, 11(7), 497-504. http://dx.doi.org/10.1038/nrmicro3050. PMid:23748339.

Kelly, C. J., Zheng, L., Campbell, E. L., Saeedi, B., Scholz, C. C., Bayless, A. J., Wilson, K. E., Glover, L. E., Kominsky, D. J., Magnuson, A., Weir, T. L., Ehrentraut, S. F., Pickel, C., Kuhn, K. A., Lanis, J. M.,
Nguyen, V., Taylor, C. T., \& Colgan, S. P. (2015). Crosstalk between microbiota-derived short-chain fatty acids and intestinal epithelial HIF augments tissue barrier function. Cell Host \& Microbe, 17(5), $662-$ 671. http://dx.doi.org/10.1016/j.chom.2015.03.005. PMid:25865369.

Kumari, R., Ahuja, V., \& Paul, J. (2013). Fluctuations in butyrate-producing bacteria in ulcerative colitis patients of North India. World Journal of Gastroenterology, 19(22), 3404-3414. http://dx.doi.org/10.3748/ wjg.v19.i22.3404. PMid:23801832.

Li, T., Qiu, Y., Yang, H. S., Li, M. Y., Zhuang, X. J., Zhang, S. H., Feng, R., Chen, B. L., He, Y., Zeng, Z. R., \& Chen, M. H. (2020). Systematic review and meta-analysis: Association of a pre-illness Western dietary pattern with the risk of developing inflammatory bowel disease. Journal of Digestive Diseases, 21(7), 362-371. http://dx.doi. org/10.1111/1751-2980.12910. PMid:32463159.

Louis, P., Hold, G. L., \& Flint, H. J. (2014). The gut microbiota, bacterial metabolites and colorectal cancer. Nature Reviews. Microbiology, 12(10), 661-672. http://dx.doi.org/10.1038/nrmicro3344. PMid:25198138.

Lozupone, C., Lladser, M. E., Knights, D., Stombaugh, J., \& Knight, R. (2011). UniFrac: an effective distance metric for microbial community comparison. The ISME Journal, 5(2), 169-172. http:// dx.doi.org/10.1038/ismej.2010.133. PMid:20827291.

Machiels, K., Joossens, M., Sabino, J., De Preter, V., Arijs, I., Eeckhaut, V., Ballet, V., Claes, K., Van Immerseel, F., Verbeke, K., Ferrante, M., Verhaegen, J., Rutgeerts, P., \& Vermeire, S. (2014). A decrease of the butyrate-producing species Roseburia hominis and Faecalibacterium prausnitzii defines dysbiosis in patients with ulcerative colitis. Gut, 63(8), 1275-1283. http://dx.doi.org/10.1136/gutjnl-2013-304833. PMid:24021287.

Magwira, C. A., Kullin, B., Lewandowski, S., Rodgers, A., Reid, S. J., \& Abratt, V. R. (2012). Diversity of faecal oxalate-degrading bacteria in black and white South African study groups: insights into understanding the rarity of urolithiasis in the black group. Journal of Applied Microbiology, 113(2), 418-428. http://dx.doi. org/10.1111/j.1365-2672.2012.05346.x. PMid:22616725.

Makki, K., Deehan, E. C., Walter, J., \& Bäckhed, F. (2018). The Impact of Dietary Fiber on Gut Microbiota in Host Health and Disease. Cell Host \& Microbe, 23(6), 705-715. http://dx.doi.org/10.1016/j. chom.2018.05.012. PMid:29902436.

Meehan, C. J., \& Beiko, R. G. (2014). A phylogenomic view of ecological specialization in the Lachnospiraceae, a family of digestive tractassociated bacteria. Genome Biology and Evolution, 6(3), 703-713. http://dx.doi.org/10.1093/gbe/evu050. PMid:24625961.

Mendes, V., Galvão, I., \& Vieira, A. T. (2019). Mechanisms by which the gut microbiota influences cytokine production and modulates host inflammatory responses. Journal of Interferon \& Cytokine Research, 39(7), 393-409. http://dx.doi.org/10.1089/jir.2019.0011. PMid:31013453.

Morgan, X. C., Tickle, T. L., Sokol, H., Gevers, D., Devaney, K. L., Ward, D. V., Reyes, J. A., Shah, S. A., LeLeiko, N., Snapper, S. B., Bousvaros, A., Korzenik, J., Sands, B. E., Xavier, R. J., \& Huttenhower, C. (2012). Dysfunction of the intestinal microbiome in inflammatory bowel disease and treatment. Genome Biology, 13(9), R79. http://dx.doi. org/10.1186/gb-2012-13-9-r79. PMid:23013615.

O’Keefe, S. J., Li, J. V., Lahti, L., Ou, J., Carbonero, F., Mohammed, K., Posma, J. M., Kinross, J., Wahl, E., Ruder, E., Vipperla, K., Naidoo, V., Mtshali, L., Tims, S., Puylaert, P. G., DeLany, J., Krasinskas, A., Benefiel, A. C., Kaseb, H. O., Newton, K., Nicholson, J. K., de Vos, W. M., Gaskins, H. R., \& Zoetendal, E. G. (2015). Fat, fibre and cancer risk in African Americans and rural Africans. Nature Communications, 6(1), 6342. http://dx.doi.org/10.1038/ncomms7342. PMid:25919227. 
Parkar, S. G., Trower, T. M., \& Stevenson, D. E. (2013). Fecal microbial metabolism of polyphenols and its effects on human gut microbiota. Anaerobe, 23, 12-19. http://dx.doi.org/10.1016/j.anaerobe.2013.07.009. PMid:23916722.

Rehman, A., Rausch, P., Wang, J., Skieceviciene, J., Kiudelis, G., Bhagalia, K., Amarapurkar, D., Kupcinskas, L., Schreiber, S., Rosenstiel, P., Baines, J. F., \& Ott, S. (2016). Geographical patterns of the standing and active human gut microbiome in health and IBD. Gut, 65(2), 238248. http://dx.doi.org/10.1136/gutjnl-2014-308341. PMid:25567118.

Rist, V. T., Weiss, E., Eklund, M., \& Mosenthin, R. (2013). Impact of dietary protein on microbiota composition and activity in the gastrointestinal tract of piglets in relation to gut health: a review. Animal, 7(7), 1067-1078. http://dx.doi.org/10.1017/S1751731113000062. PMid:23410993.

Scarmozzino, F., Poli, A., \& Visioli, F. (2020). Microbiota and cardiovascular disease risk: a scoping review. Pharmacological Research, 159, 104952. http://dx.doi.org/10.1016/j.phrs.2020.104952. PMid:32492487.

Schloss, P. D., Gevers, D., \& Westcott, S. L. (2011). Reducing the effects of PCR amplification and sequencing artifacts on $16 \mathrm{~S}$ rRNA-based studies. PLoS One, 6(12), e27310. http://dx.doi.org/10.1371/journal. pone.0027310. PMid:22194782.

Segata, N., Izard, J., Waldron, L., Gevers, D., Miropolsky, L., Garrett, W. S., \& Huttenhower, C. (2011). Metagenomic biomarker discovery and explanation. Genome Biology, 12(6), R60. http://dx.doi.org/10.1186/ gb-2011-12-6-r60. PMid:21702898.

Sokol, H., Pigneur, B., Watterlot, L., Lakhdari, O., Bermúdez-Humarán, L. G., Gratadoux, J. J., Blugeon, S., Bridonneau, C., Furet, J. P., Corthier, G., Grangette, C., Vasquez, N., Pochart, P., Trugnan, G., Thomas, G., Blottière, H. M., Doré, J., Marteau, P., Seksik, P., \& Langella, P. (2008). Faecalibacterium prausnitzii is an anti-inflammatory commensal bacterium identified by gut microbiota analysis of Crohn disease patients. Proceedings of the National Academy of Sciences of the United States of America, 105(43), 16731-16736. http://dx.doi. org/10.1073/pnas.0804812105. PMid:18936492.

Steer, T., Carpenter, H., Tuohy, K., \& Gibson, G. R. (2000). Perspectives on the role of the human gut microbiota and its modulation by pro- and prebiotics. Nutrition Research Reviews, 13(2), 229-254. http://dx.doi.org/10.1079/095442200108729089. PMid:19087441.

Huang, T., Yang, X., Ji, J., Wang, Q., Wang, H., \& Dong, Z. (2020). Inhibitory effects of tanshinone IIA from Salvia miltiorrhiza Bge on human bladder cancer BIU-87 cells and xenograft in nude mice. Food Science and Technology (Campinas), 40(1), 209-214. http:// dx.doi.org/10.1590/fst.38818.

van Hylckama Vlieg, J. E., Veiga, P., Zhang, C., Derrien, M., \& Zhao, L. (2011). Impact of microbial transformation of food on health from fermented foods to fermentation in the gastro-intestinal tract.
Current Opinion in Biotechnology, 22(2), 211-219. http://dx.doi. org/10.1016/j.copbio.2010.12.004. PMid:21247750.

Varela, E., Manichanh, C., Gallart, M., Torrejón, A., Borruel, N., Casellas, F., Guarner, F., \& Antolin, M. (2013). Colonisation by Faecalibacterium prausnitzii and maintenance of clinical remission in patients with ulcerative colitis. Alimentary Pharmacology \& Therapeutics, 38(2), 151-161. http://dx.doi.org/10.1111/apt.12365. PMid:23725320.

Wang, Q., Hao, C., Yao, W., Zhu, D., Lu, H., Li, L., Ma, B., Sun, B., Xue, D., \& Zhang, W. (2020). Intestinal flora imbalance affects bile acid metabolism and is associated with gallstone formation. $B M C$ Gastroenterology, 20(1), 59. http://dx.doi.org/10.1186/s12876-02001195-1. PMid:32143645.

Wu, G. D., Chen, J., Hoffmann, C., Bittinger, K., Chen, Y. Y., Keilbaugh, S. A., Bewtra, M., Knights, D., Walters, W. A., Knight, R., Sinha, R., Gilroy, E., Gupta, K., Baldassano, R., Nessel, L., Li, H., Bushman, F. D., \& Lewis, J. D. (2011). Linking long-term dietary patterns with gut microbial enterotypes. Science, 334(6052), 105-108. http://dx.doi. org/10.1126/science.1208344. PMid:21885731.

Yang, J., Cui, J., Han, H., Chen, J., Yao, J., \& Liu, Y. (2020). Determination of active compounds in raspberry leaf extracts and the effects of extract intake on mice. Food Science and Technology (Campinas), 40(1), 124-131. http://dx.doi.org/10.1590/fst.35518.

Yatsunenko, T., Rey, F. E., Manary, M. J., Trehan, I., Dominguez-Bello, M. G., Contreras, M., Magris, M., Hidalgo, G., Baldassano, R. N., Anokhin, A. P., Heath, A. C., Warner, B., Reeder, J., Kuczynski, J., Caporaso, J. G., Lozupone, C. A., Lauber, C., Clemente, J. C., Knights, D., Knight, R., \& Gordon, J. I. (2012). Human gut microbiome viewed across age and geography. Nature, 486(7402), 222-227. http://dx.doi. org/10.1038/nature11053. PMid:22699611.

Zhang, Z., Wang, X., Han, S., Liu, C., \& Liu, F. (2018). Effect of two seaweed polysaccharides on intestinal microbiota in mice evaluated by illumina PE250 sequencing. International Journal of Biological Macromolecules, 112, 796-802. http://dx.doi.org/10.1016/j. ijbiomac.2018.01.192. PMid:29427682.

Zhong, V. W., Van Horn, L., Greenland, P., Carnethon, M. R., Ning, H., Wilkins, J. T., Lloyd-Jones, D. M., \& Allen, N. B. (2020). Associations of processed meat, unprocessed red meat, poultry, or fish intake with incident cardiovascular disease and all-cause mortality. JAMA Internal Medicine, 180(4), 503-512. http://dx.doi.org/10.1001/ jamainternmed.2019.6969. PMid:32011623.

Zimmer, J., Lange, B., Frick, J. S., Sauer, H., Zimmermann, K., Schwiertz, A., Rusch, K., Klosterhalfen, S., \& Enck, P. (2012). A vegan or vegetarian diet substantially alters the human colonic faecal microbiota. European Journal of Clinical Nutrition, 66(1), 53-60. http://dx.doi.org/10.1038/ejcn.2011.141. PMid:21811294. 